\title{
PREVALÊNCIA DE CÂNCER DE MAMA E ASSOCIAÇÃO COM SEUS FATORES PROGNÓSTICOS E PREDITIVOS
}

\author{
Ariane Maria Peres T. de Almeida ${ }^{1}$, Hugo Ricardo Marquini ${ }^{1}$, Rafaela Mendonça Leite ${ }^{1}$, Gisele Alborghetti \\ $\mathrm{Nai}^{2}$ \\ ${ }^{1}$ Discente da Faculdade de Medicina de Presidente Prudente (FMPP) da UNOESTE; ${ }^{2}$ Médica patologista, Docente do Departamento de Patologia, \\ responsável técnica pelo Laboratório de Anatomia Patológica da UNOESTE, Presidente Prudente, SP.
}

\section{RESUMO}

O câncer de mama é o tipo de câncer mais frequente em mulheres no Brasil. O câncer de mama tem um prognóstico relativamente bom, se diagnosticado e tratado oportunamente. Porém, as taxas de mortalidade para este tipo de câncer continuam elevadas no Brasil, muito provavelmente porque a doença ainda é diagnosticada em estágios avançados. O objetivo do presente estudo foi avaliar a prevalência de câncer de mama por meio do exame anátomo-patológico e os fatores prognósticos e preditivos associados a cada caso, para observar o estágio de diagnóstico das pacientes. Foi realizado um estudo retrospectivo com seleção dos laudos anatomo-patológicos de exérese de tumor mamário com diagnóstico de câncer de mama, provenientes do Hospital Universitário de Presidente Prudente - SP, realizados no período de 1998 a 2009. Dos laudos de exame foram retirados os dados relacionados à idade da paciente, os fatores prognósticos e os fatores preditivos. Foram encontrados 183 casos de câncer de mama. A maior prevalência ocorreu na faixa etária entre 40 a 49 anos (29,5\%) e o tipo histológico mais frequente foi o Carcinoma ductal invasivo (61,2\%). Em $45 \%$ dos casos, os tumores mediram até $2 \mathrm{~cm}$. A maioria (71\%) não apresentava metástases para os linfonodos axilares. Em 27 casos houve expressão de receptores hormonais e em 26 hiperexpressão de Her2/neu. Conclui-se que as pacientes estudadas foram diagnosticadas na fase inicial do câncer de mama.

Palavras-chave: Neoplasias da mama, diagnóstico, prognóstico, patologia, estágio clínico.

\section{PREVALENCE OF BREAST CANCER AND ITS ASSOCIATION WITH PROGNOSTIC AND PREDICTIVE FACTORS}

\begin{abstract}
Breast cancer is the most frequent cancer in women in Brazil. Although it is considered a cancer with relatively good prognosis, if diagnosed and treated appropriately, mortality rates from breast cancer remain high in Brazil, most likely because the disease is still diagnosed in advanced stages. The aim of this study was to evaluate the prevalence of breast cancer in a university hospital by pathological examination and the prognostic and predictive factors associated with each case, to observe the stage of diagnosis of patients. We conducted a retrospective study with selection of anatomic-pathological reports of mammary tumor excision diagnosed with breast cancer, from the University Hospital of Presidente Prudente - SP, in the period from 1998 to 2009 . We collected the data related to patient age and prognostic and predictors factors. There were 183 cases of breast cancer. The highest incidence occurred between the ages of 40-49 years $(29.5 \%)$ and the most frequent histological type was invasive ductal carcinoma (61.2\%). In $45 \%$ of cases, the tumors were measured less than $2 \mathrm{~cm}$. The majority (71\%) had no metastasis to axillary lymph nodes. In 27 cases there was expression of hormone receptors and in 26 patients were overexpression of Her2/neu. It is concluded that the patients studied were diagnosed in early stage of breast cancer.
\end{abstract}

Keywords: Breast neoplasms, diagnosis, prognosis, pathology, clinical clerkship. 


\section{INTRODUÇÃO}

O câncer de mama é a principal neoplasia maligna que acomete o sexo feminino no Brasil e no mundo. Em 2003, apresentou incidência bruta estimada de 46,35 casos por 100 mil mulheres (SCLOWITZ et al., 2005). O número de casos novos de câncer de mama esperados para o Brasil em 2010 foi de 49.240, com um risco estimado de 49 casos a cada 100 mil mulheres e em 2012, esperam-se 52.680 casos novos de câncer da mama, com um risco estimado de 52 casos a cada 100 mil mulheres (BRASIL, 2011). Assim, pode-se observar uma incidência crescente dos casos novos de câncer de mama ao longo desta década.

O diagnóstico precoce do câncer de mama tem importância fundamental para 0 tratamento e prognóstico da doença, pois permite a realização de cirurgias menos mutilantes, com menor morbidade, como a retirada apenas do nódulo, com possibilidade de cura total, além de proporcionarem menores gastos hospitalares (NAl et al., 2009). Cânceres de mama não palpáveis são aqueles detectados, exclusivamente, por mamografia e ultra-som, exames estes que permitem o diagnóstico precoce do tumor (SCLOWITZ et al., 2005; NAl et al., 2006). Porém, o medo do diagnóstico de câncer e a possível necessidade da retirada da mama levam muitas mulheres a não realizarem a mamografia (NAl et al., 2006).

Os fatores prognósticos e preditivos podem ser usados para indicar a presença, o estado e o futuro comportamento e também a resposta da mulher com câncer de mama a várias terapias (WILLIANS et al., 2006; SONG et al., 2011).

Fatores prognósticos são parâmetros possíveis de serem mensurados no momento do diagnóstico e que servem como preditor da sobrevida do paciente (PAULINELLI et al., 2003).
Os fatores prognósticos para o câncer de mama são: o tamanho tumoral, grau histológico do tumor, invasão vascular, índice mitótico metástase para linfonodos, estado do receptor de estrógeno e alteração na família dos receptores para os fatores de crescimento epidérmico (Her2, CerbB2), (WILLIANS et al., 2006, SONG et al., 2011).

Fatores preditivos são aqueles que determinam a resposta a uma terapêutica específica para um tipo de câncer (WILLIANS et al., 2006). O receptor de estrógeno é de fundamental importância no câncer de mama, por levar a proliferação de suas células tumorais e por ser alvo terapêutico para inibição do crescimento tumoral. Além disso, o HER2/neu, um receptor para fator de crescimento pode ser inibido por medicamentos ou anticorpos e também é um alvo importante para terapia no câncer de mama (WILLIANS et al., 2006).

O objetivo do presente estudo foi avaliar a prevalência de câncer de mama utilizando o exame anátomo-patológico e os fatores prognósticos e preditivos associados a cada caso, para observar o estágio de diagnóstico das pacientes.

\section{MÉTODO}

Foi realizado um estudo retrospectivo com seleção dos laudos anatomo-patológicos de exérese de tumor mamário com diagnóstico de câncer de mama, provenientes do Hospital Universitário de Presidente Prudente - SP, realizados no período de 1998 a 2009 (período de funcionamento deste hospital como universitário). Este estudo foi aprovado pelo Comitê de Ética em Pesquisa da Universidade do Oeste Paulista (CEP/UNOESTE - Processo n 927/2011).

Foram excluídos os casos de biópsia incisional ou biópsias por agulha grossa (core 
biopsy), que não permitem a avaliação de todos os parâmetros prognósticos.

Dos laudos de exame foram obtidos os dados relacionados à idade da paciente, os fatores prognósticos (tipo histológico do tumor, tamanho do tumor, grau histológico do tumor, presença de invasão angio-linfática, índice mitótico e presença de metástase para linfonodos) e os fatores preditivos (pesquisa de receptores de estrógeno e progesterona e da oncoproteína Her2/neu por meio do exame imunoistoquímico complementar).

Para a análise estatística utilizou-se o teste do qui-quadrado ao nível de significância de $p<0,05$. Todas as variáveis prognósticas (tipo histológico do tumor, tamanho do tumor, grau histológico do tumor, presença de invasão angiolinfática, índice mitótico e presença de metástase para linfonodos) e as preditivas (pesquisa de receptores de estrógeno e progesterona e da oncoproteína Her2/neu) foram comparadas umas com as outras. Os testes estatísticos foram realizados com o pacote SPSS - Statistical Package for the Social Sciences v.15.0.

\section{RESULTADOS}

Foram encontrados 183 laudos anatomopatológicos de exérese de câncer de mama no período de 1998 a 2009. A idade das pacientes variou entre 22 e 87 anos, com maior incidência entre 40-59 anos (47,5\%), média de 52,9 anos (desvio padrão - DP de 14,7 anos) e mediana de 50 anos. $O$ tamanho dos tumores variou entre 1 a $14 \mathrm{~cm}$, sendo que a maioria (45\%) media até $2 \mathrm{~cm}$.

Dos 183 casos, 112 foram diagnosticados como carcinoma ductal invasivo, 40 casos de carcinoma ductal in situ, 17 casos de carcinoma lobular invasivo, três casos de carcinoma colóide invasivo, dois casos cada de carcinoma apócrino invasivo e de carcinoma lobular in situ, um caso cada de doença de Paget, de carcinoma papilífero, de carcinoma sarcomatóide, de carcinoma tubular invasivo, de neoplasia maligna anaplásica e de neoplasia maligna indiferenciada.

A maioria das pacientes tinha grau histológico II (33,8\%), seguida pelas pacientes com grau histológico I (30\%). Dentre os tumores com grau I histológico, 52,7\% mediam menos de $2 \mathrm{~cm}$, dos tumores com grau II histológico, 29\% media menos de $2 \mathrm{~cm}$ e $48,4 \%$ mais de $2 \mathrm{~cm}$ e daqueles com grau III, 56,5\% media mais de 2 $\mathrm{cm}$.

O índice mitótico variou de 0 a 15 mitoses por 10 campos de grande aumento (CGA), com média de 4,2 mitoses/10CGA ( $D P=3,3$ mitoses/10CGA) e mediana de 3 mitoses/10CGA. Muitos casos não tinham descrito o índice mitótico ( $n=126)$.

Somente o carcinoma ductal invasivo, o lobular invasivo e o sarcomatóide apresentaram índices mitóticos maiores que 6 mitoses/10CGA $(p<0,05)$. Todos os carcinomas in situ apresentaram índice mitótico menor que 5 mitoses/10CGA (Figura 1).

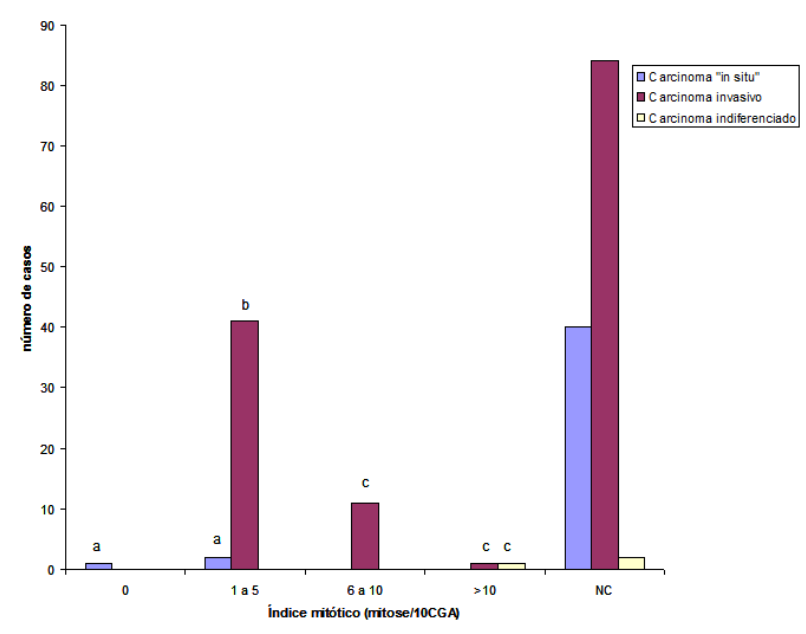

Figura 1. Distribuição das pacientes comparandose o tipo histológico e índice mitótico do câncer de mama ( $n=183)$. NC - não contém. Barras com diferentes sobrescritos diferem significativamente $(p<0,05)$. 
Somente os tumores com grau histológico III apresentaram mais de 10 mitoses/10CGA. Índice mitótico 0 (zero) ocorreu em apenas um caso de tumor de grau histológico I $(p<0,05)$.

Em 129 casos (70,5\%), não houve invasão angio-linfática do tumor ao exame histopatológico. Os carcinomas invasivos independente do subtipo histológico apresentaram invasão angio-linfática em 33 casos $(24,1 \%) \quad(p<0,05)$. Não houve associação estatisticamente significante entre o grau histológico do tumor e invasão angio linfática $(p>0,05)$. O maior índice de invasão angiolinfática foi entre os tumores maiores que $2 \mathrm{~cm}$ $(66,8 \%) \quad(p<0,05)$. A maioria dos casos $(n=98)$ sem invasão angio-linfática não apresentava o índice mitótico descrito, porém nos 31 casos que continham este dado, a maioria $(80,6 \% ; n=25)$ apresentava índice mitótico menor que 6 mitoses/10CGA $(p<0,05)$.

Em relação à disseminação do tumor, a maioria dos casos (71\%) não apresentou metástase do câncer de mama para linfonodos axilares e destes $86,8 \% \quad(n=112)$ não apresentavam invasão angio-linfática $(p<0,05)$. 0 nível I foi o mais comprometido, em $86,8 \%$ dos casos com comprometimento linfonodal e o nível III em $26,3 \%$ destes $(p<0,05)$. O número de linfonodos comprometidos nos níveis I, II e III variou, respectivamente, entre 1 a 22 (mediana de 3), 1 a 10 (mediana de 2) e 1 a 6 (mediana de 2). Somente em quatro casos houve avaliação do linfonodo sentinela, os quais não apresentavam metástase do câncer de mama.

O carcinoma ductal invasivo foi o que apresentou maior porcentagem de metástase para linfonodos $(76,3 \%)$, assim como os carcinomas com medidas maiores que $2 \mathrm{~cm}$ $(57,9 \%)$ (Figura 2) e grau II histológico $(55,3 \%)$ (Figura 3) $(p<0,05)$. Em relação ao índice mitótico não houve relação estatisticamente significante com metástase para linfonodos $(p>0,05)$.

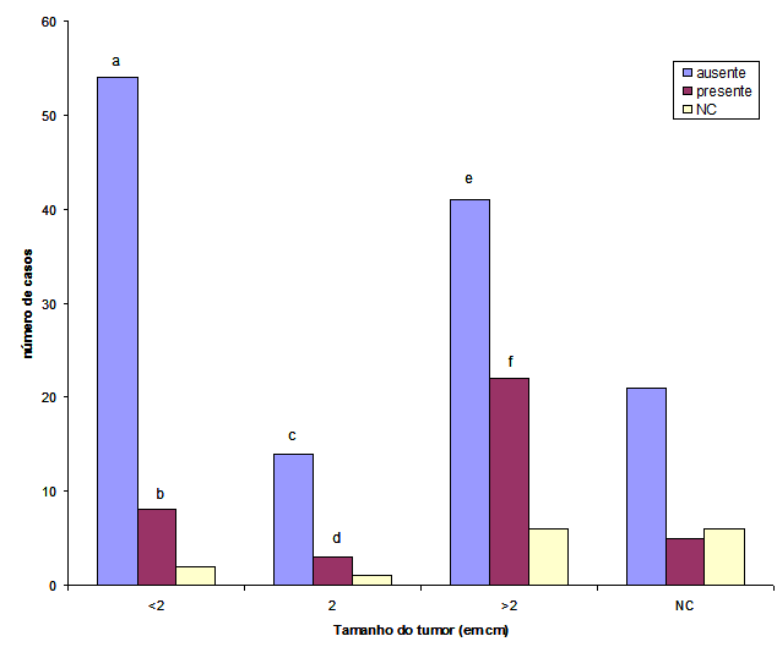

Figura 2. Distribuição das pacientes comparandose o tamanho do tumor e metástase para os linfonodos axilares $(n=183)$. NC - não contém. Barras com diferentes sobrescritos diferem significativamente $(p<0,05)$.

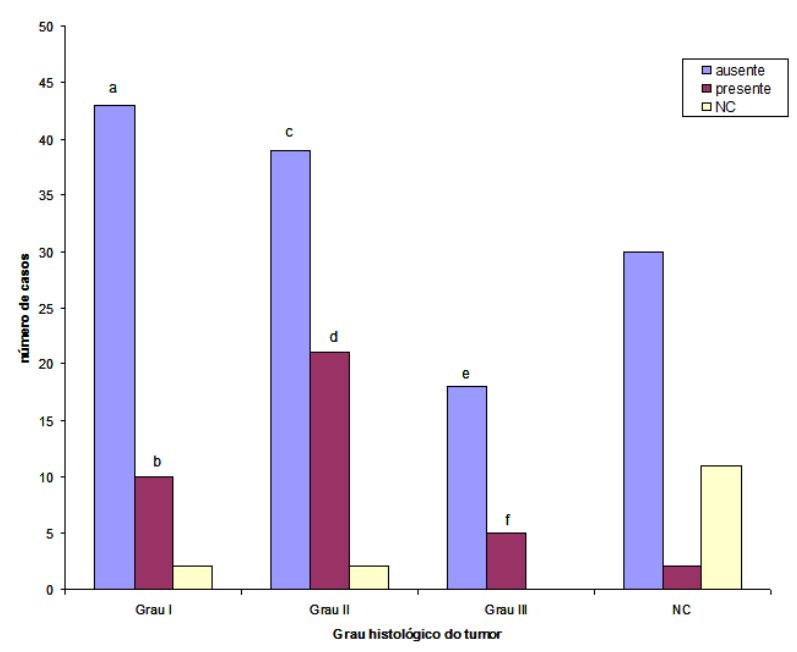

Figura 3. Distribuição das pacientes comparandose o grau histológico e metástase para os linfonodos axilares $(n=183)$. Barras com diferentes sobrescritos diferem significativamente $(p<0,05)$.

Conseguiu-se obter os dados sobre a pesquisa dos receptores hormonais (estrógeno e progesterona) e da hiperexpressão da oncoproteína Her2/neu no material de biópsia em 38 e 39 casos, respectivamente. Destes, $71,8 \%$ apresentava expressão de receptores hormonais nas células tumorais e $66,6 \%$ hiperexpressão de Her2/neu $(\mathrm{p}<0,05)$. A Tabela 1 abaixo compara a positividade dos receptores hormonais e a 
hiperexpressão de Her2/neu nos casos estudados.

Tabela 1. Comparação entre a positividade dos receptores hormonais e a hiperxpressão de Her2/neu nos casos estudados de câncer de mama ( $n=183)$.

\begin{tabular}{lcccc}
\hline \multirow{2}{*}{ Her2/neu } & \multicolumn{2}{c}{ Receptor de estrógeno e progesterona } & \multirow{2}{*}{ Total } \\
\cline { 2 - 4 } & positivo & negativo & NC & \\
\hline Hiperexpresso & $19^{\mathrm{a}}$ & $7^{\mathrm{b}}$ & 0 & 26 \\
Sem hiperexpressão & $8^{\mathrm{c}}$ & $4^{\mathrm{a}}$ & 1 & 13 \\
NC & 0 & 0 & 144 & 144 \\
Total & 27 & 11 & 145 & 183 \\
\hline
\end{tabular}

NC - não contém. Diferentes sobrescritos indicam diferença significaticante $(p<0,05)$.

Dos 33 casos de carcinoma invasivo onde houve a pesquisa de receptores de estrógeno e progesterona (RE/RP), esta foi positiva em $69,7 \%$ destes. No caso da pesquisa de Her2/neu em 34 casos de carcinomas invasivos, este apresentou hiperexpressão em 61,7\%.

O grau II histológico foi o que apresentou maior positividade para RE/RP $(40,7 \%)$ e hiperexpressão para Her2/neu $(42,3 \%)(p<0,05)$. Porém, em relação ao tamanho do tumor, a positividade para RE/RP foi maior entre os tumores menores que $2 \mathrm{~cm} \quad(33,3 \%)$ e a hiperexpressão de Her2/neu foi mais evidente nos tumores maiores que $2 \mathrm{~cm}(34,6 \%)(p<0,05)$.

Não houve relação estatisticamente significante tanto para a positividade para RE/RP, quanto para hiperexpressão de Her2/neu e índice mitótico $(p>0,05)$.

Em dezoito casos, onde se identificou invasão angio-linfática, ocorreu positividade para RE/RP (47,3\%) e em apenas cinco, hiperexpressão de Her2/neu $(12,8 \%)(p<0,05)$.

$\mathrm{Na}$ metade dos casos com positividade para RE/RP e em 46,1\% daqueles com hiperexpressão de Her2/neu não ocorreu metástase para linfonodos axilares $(p<0,05)$.

\section{DISCUSSÃO}

Neste estudo, a faixa etária das pacientes que ocorreu a maior prevalência de câncer de mama (40 a 59 anos) foi semelhante ao que é descrito na literatura (SCLOWITZ et al., 2005). O que corrobora as recomendações do Ministério da Saúde para detecção precoce e diagnóstico desse câncer baseadas no Documento de Consenso para Controle do Câncer de Mama, de 2004, que considera como principais estratégias de rastreamento um exame mamográfico, pelo menos a cada dois anos, para mulheres entre 50 a 69 anos, e o exame clínico anual das mamas, para mulheres de 40 a 49 anos (BRASIL, 2004).

A literatura mostra que há uma associação estatisticamente significante entre o tamanho do tumor e tempo de vida livre de doença, tempo de vida global e ambos (WILLIANS et al., 2006). No presente estudo, a maioria do tumores (45\%) media até $2 \mathrm{~cm}$, mostrando um diagnóstico precoce do tumor e assim melhor prognóstico para a paciente.

Aproximadamente $90 \%$ de todos os cânceres de mama ocorrem nos ductos ou nos lóbulos, $80 \%$ de todos os casos iniciam nas células de revestimento dos ductos mamários. Este tipo de câncer é chamado de carcinoma ductal. O câncer que inicia nos lóbulos é chamado de carcinoma lobular e é bilateral em 
$30 \%$ dos casos (WILLIANS et al., 2006). Se a doença rompe o ducto e atinge os tecidos em volta, é chamado de infiltrativo, invasivo ou invasor, podendo ser: carcinoma ductal invasor ou carcinoma lobular invasor. A doença que está contida dentro dos ductos é chamada de in situ. Outros tipos de câncer de mama menos frequentes são: tipo medular, mucinoso, tubular e papilar, correspondendo a menos de $10 \%$ de todos os casos e, apresentando melhor prognóstico (WILLIANS et al., 2006; BROT et al., 2009). Este estudo corrobora com a literatura em relação à frequência dos tipos histológicos (WILLIANS et al., 2006), pois dos 183 casos estudados, 112 foram diagnosticados como carcinoma ductal invasivo, 40 casos de carcinoma ductal in situ, 17 casos de carcinoma lobular invasivo.

A avaliação histológica das seguintes características - formação de túbulos, atipias nucleares e índice mitótico - serve para graduar os carcinomas em: bem diferenciado (grau I), moderadamente diferenciado (grau II) e pouco diferenciado (grau III) (WILLIANS et al., 2006). O grau histológico do tumor é um dos mais consistentes fatores prognósticos para guiar os tratamentos adjuvantes nos carcinomas invasivos da mama e é usado na maioria dos algoritmos de decisão de tratamento atuais (ELSTON; ELLIS, 2002; REYAL et al., 2012). Neste trabalho, a maioria das pacientes tinha grau histológico II (34\%), seguida pelas pacientes com grau histológico I (30\%), mostrando que em grande número de casos o tumor era menos agressivo. Este fato é confirmado quando se compara o grau histológico ao tamanho dos tumores, pois a maioria dos tumores com grau I histológico $(52,7 \%)$ media menos de $2 \mathrm{~cm}$, e a maioria dos tumores com grau II histológico $(48,4 \%)$ e grau III $(56,5 \%)$ media mais de $2 \mathrm{~cm}$. A literatura relata que cerca de 40 a $50 \%$ dos tumores pequenos e sem metástase para linfonodos são grau II histológico (REYAL et al., 2012), neste estudo $29 \%$ dos tumores grau II media menos de $2 \mathrm{~cm}$.

Há muitos fatores de crescimento e receptores envolvidos no câncer de mama, mas a via final comum destes, a proliferação celular, pode ser medida. A proliferação celular pode ser acessada pela contagem de células se dividindo (índice mitótico) ou pela expressão de moléculas chaves envolvidas na regulação do ciclo celular (WILLIANS et al., 2006). A taxa de proliferação celular é o maior fator de resposta à quimioterapia (REYAL et al., 2012).

O índice mitótico é definido como o número de mitoses por 10 campos de grande aumento (CGA) na periferia do tumor. Este índice está ligado a ambos, a porcentagem de células tumorais em mitose e a duração do ciclo celular, considerando que a fase $M$ é somente parte do processo do ciclo celular (REYAL et al., 2012).

Um escore de índice mitótico de 3 (mais de 21 mitoses/10CGA) apresenta um risco relativo de 4,4 comparado com aquelas com um escore de 1 (menos de 10 mitoses/10GGA) para tempo livre de doença por 5 anos (WILLIANS et al., 2006). No presente trabalho, o índice mitótico dos tumores variou de 0 a 15 mitoses/10 CGA, com média de 4,2 mitoses/10CGA, mostrando que a maioria dos casos tinha escore 1 , o que resulta num melhor prognóstico para estas pacientes.

Os tipos histológicos mais agressivos, como o carcinoma ductal, sacomatóide, anaplásico e os indiferenciados estão associados a índices mitóticos mais elevados (WILLIANS et al., 2006). Neste estudo, muitos casos não tinham descrito o índice mitótico $(n=126)$, o que prejudica a avaliação, mas pode-se observar que somente o carcinoma ductal invasivo, o lobular e o anaplásico apresentaram índice mitótico maior que 6 mitoses/10CGA.

O grau histológico está muito relacionado ao índice mitótico e este último é um dos 
parâmetros usados para se fazer a graduação (REYAL et al., 2012). Neste estudo, somente os tumores com grau histológico 3 apresentaram mais de 10 mitoses/10CGA e índice mitótico 0, somente um caso de tumor de grau histológico 1.

Para as células tumorais se disseminarem do tumor primário para locais a distância (metástases) é necessário que ocorra destruição da matriz extracelular ao redor destas células. Para que ocorra metástase, inicialmente as células tumorais precisam invadir os vasos sanguíneos locais (invasão vascular) ou os vasos linfáticos (invasão linfática), o que é frequentemente relatado como invasão angiolinfática (WILLIANS et al., 2006). Assim, a invasão angio-linfática é definida como um êmbolo tumoral presente entre um espaço endotelial bem definido na mama ao redor do carcinoma invasivo (SONG et al., 2011). Na maioria dos casos estudados (70,5\%) não houve invasão angio-linfática do tumor ao exame histopatológico e os carcinomas invasivos independente do subtipo histológico apresentaram invasão angio-linfática em 24,1\% dos casos, corroborando outros dados que favorecem o diagnóstico precoce do câncer de mama destas pacientes. Não se observou associação estatisticamente significante entre o grau histológico do tumor e invasão angiolinfática, talvez por que a maioria das pacientes apresentava grau histológico I ou II. O maior índice de invasão angio-linfática foi entre os tumores maiores que $2 \mathrm{~cm}$ (66,8\%), o que confirma o descrito na literatura onde tumores maiores tem maior risco de disseminação (WILLIANS et al., 2006). A maioria dos casos com descrição do índice mitótico (80,6\%; $n=25)$ apresentava menos de 6 mitoses/10CGA, o que mostra que não somente a proliferação celular é um fator de risco para invasão vascular.

As células do câncer de mama podem migrar para os vasos sanguíneos e vasos linfáticos e linfonodos. Os linfonodos podem se localizar na região axilar, região supra-clavicular, região cervical e mamária interna. Aproximadamente, 95\% da drenagem linfática da mama se dirigem para os linfonodos axilares (WILLIANS et al., 2006). O status dos linfonodos axilares é um fortíssimo fator prognóstico em pacientes com tumores primários mamários operáveis (FANEYTE et al., 2004; SONG et al., 2011). Além disso, a positividade dos linfonodos parece ser o único fator clínico mais importante para indicar quimioterapia adjuvante (WILLIANS et al., 2006). Comparando-se pacientes com câncer de mama sem envolvimento axilar e aquelas com linfonodos positivos a sobrevida de 10 anos, vai de 65 a $80 \%$ para 25 a 48\%. O prognóstico piora com o aumento do número de linfonodos envolvidos. Pacientes com quatro ou mais linfonodos positivos entram para um subgrupo com prognóstico desfavorável (FANEYTE et al., 2004). Neste estudo, 71\% $(n=130)$ dos casos não apresentaram metástase do câncer de mama para linfonodos axilares e a mediana de comprometimento foi de três linfonodos no nível I e dois nos níveis II e III. Dados estes, que comprovam o diagnóstico precoce e prognóstico favorável das pacientes estudadas.

A existência de invasão angio-linfática pode ajudar a identificar quem tem risco aumentado para metástase para linfonodos axilares ou à distância. Embora a invasão angiolinfática tem sido aceita como um importante fator prognóstico nas pacientes com câncer de mama invasivo linfonodo-negativo, ela é controversa em relação ao valor prognóstico em pacientes com linfonodos positivos (SONG et al., 2011). Dos casos linfonodos-negativos deste estudo, 86,8\% (n=112) não apresentavam invasão angio-linfática associada.

Assim como no caso da presença de invasão angio-linfática, o carcinoma ductal 
invasivo foi o que apresentou maior porcentagem de metástase para linfonodos (76,3\%), assim como os carcinomas com medidas maiores que 2 cm (57,9\%). Porém aqui houve correlação com o grau histológico, onde o grau II mostrou metástase na maioria dos casos (55,3\%), mas não com o índice mitótico. Estes dados mostram que tumores maiores, de maior grau e de tipo histológico mais agressivo têm risco maior de metástase para linfonodos como descrito na literatura (RIVADENEIRA et al., 2000). No caso com a correlação com o índice mitótico, a avaliação deste dado pode ter sido prejudicada, pois em muitos casos, este não estava descrito no laudo.

O lindonodo sentinela é o primeiro linfonodo a receber células provenientes do tumor primário através da circulação dos vasos linfáticos. O exame do linfonodo sentinela é capaz de informar com alto grau de certeza o estado dos outros linfonodos da região linfática estudada (axiliar, inguinal) (TAKEl et al., 2007). A biópsia do linfonodo sentinela é um cuidado padrão para pacientes em estágio inicial do câncer de mama e a dissecção dos linfonodos axilares é considerada desnecessária se quando o linfonodo sentinela é considerado livre de tumor (TAKEl et al., 2007; GATEK et al., 2012). Segundo a American Society of Clinical Oncology (ASCO), não está indicada a biópsia de linfonodo sentinela nos seguintes casos: tumores estádio T3 e T4, câncer inflamatório mamário, carcinoma ductal in situ sem mastectomia, linfonodos axilares suspeitos para metástase, gravidez, cirurgia axilar prévia, cirurgia mamária não oncológica prévia e após terapia preoperatória sistêmica (TAKEl et al., 2007). Embora, a maioria das pacientes estudadas apresentasse tumores em estágios iniciais, somente em quatro casos foram realizadas biópsias do linfonodo sentinela. Os autores deste estudo não tiveram acesso aos prontuários das pacientes para saber o motivo pelo qual não foi realizada a biópsia do linfonodo sentinela nos outros casos.

A análise dos receptores hormonais deve ser realizada em todos os tumores primários de mama, preferencialmente por imuno-histoquímica, por constituir um importante fator prognóstico e preditivo, constituindo um guia para a terapêutica hormonal. Pacientes com tumores RE-positivos apresentam intervalos livres da doença mais longos que as pacientes com tumores REnegativos: tumores RE-positivos apresentam intervalo livre de doença de $74 \%$ e sobrevida geral de 92\% em cinco anos, enquanto tumores RE-negativos apresentam intervalo livre de doença e sobrevida geral de $66 \%$ e $82 \%$, respectivamente (PAYNE et al., 2008). Alguns estudos apontam que a agressividade dos tumores se deve ao fato de estarem relacionados ao RE positivo ou negativo. As variações morfológicas também estão relacionadas ao RE, como por exemplo, os carcinomas medulares são RE-negativos e os carcinomas tubulares e lobulares são RE-positivos (VIEIRA et al., 2008). O estado do RP (receptor de progesterona) está mais associado à sobrevida total, mas o seu valor está menos estabelecido (PAYNE et al., 2008; RASTELLI e CRISPINO, 2008). A pesquisa de RE e RP é rotineiramente usada para selecionar as pacientes que receberão ou não terapia homonal (ex. tamoxifeno). Há muitos dados na literatura a favor da terapia adjuvante não só na doença precoce como no tratamento da doença avançada e/ou metastática (WILLIANS et al., 2006; KUROSUMI, 2003). Neste estudo, foi possível se obter os dados sobre a pesquisa de receptores de RE/RP em apenas 38 casos e destes a maioria foi positiva para ambos (71\%).

Neste estudo, na maioria dos casos de carcinoma invasivo onde houve a pesquisa de receptores de RE/RP, esta foi positiva, assim como o grau II histológico foi o que apresentou maior positividade para RE/RP (40,7\%). Porém, 
em relação ao tamanho do tumor, a positividade para RE/RP foi maior entre os tumores menores que $2 \mathrm{~cm}(33,3 \%)$ e não houve relação estatisticamente significante tanto para a positividade para RE/RP e índice mitótico. Estes dados concordam com os dados de literatura, onde é descrito que os níveis de RE e RP estão forte e inversamente relacionados com medidas de proliferação e diretamente relacionados com o grau histológico (KUROSUMI, 2003).

A presença de RE ou RP é um poderoso fator preditivo de benefício ao tratamento adjuvante com tamoxifeno, significando uma redução proporcional do risco de recorrência e mortalidade em cinco anos de $47 \%$ e $26 \%$, respectivamente. Cinco anos de tamoxifeno adjuvante também representa uma redução proporcional de $47 \%$ no risco de câncer de mama contralateral. A redução proporcional da mortalidade foi similar para pacientes com linfonodos-negativos e linfonodos-positivos (RASTELLI; CRISPINO, 2008). Em dezoito casos, onde se identificou invasão angio-linfática, ocorreu positividade para RE/RP, porém na metade dos casos com positividade para RE/RP, os linfonodos axilares foram negativos. Isto mostra que o tratamento adjuvante para estas pacientes seria de grande benefício.

O proto-oncogene Her-2/neu (c-erbB-2) está localizado no cromossomo 17q21 e codifica uma glicoproteína transmembrana, p185HER2, que tem importante atividade na regulação do crescimento celular. A proteína HER-2/neu é expressa em níveis baixos nas células epiteliais e mioepiteliais do tecido mamário normal. O gene está amplificado e/ou hiperexpresso em aproximadamente 20 a 30\% dos tumores de mama (JIANG et al., 2012). Sua amplificação e hiperexpressão estão associadas ao pior prognóstico e resistência a drogas citotóxicas em pacientes com câncer de mama. A amplificação do gene Her2 tem sido usada para predizer não somente o prognóstico, mas também para guiar o tratamento de carcinoma ductal invasivo da mama com trastuzumab. A correta avaliação do status do Her2 é importante para o manejo de pacientes candidatas a terapias que têm como alvo o Her2 (JIANG et al., 2012). Na doença metastática, a presença de hiperexpressão de HER2/neu é um preditor de resposta ao anticorpo humanizado, trastuzumab, com taxas de resposta de $11,6 \%$ com remissão completa, sem progressão do tumor por mais de 24 meses e resposta mínima ou doença estável em 37\% das pacientes (JIANG et al., 2012). A hiperexpressão da proteína Her2 pode ser estabelecida mensurando-se a expressão desta através de estudo imuno-histoquímico (WILLIANS et al., 2006). Neste estudo, conseguiram-se dados relativos à pesquisa de Her2/neu pela imunohistoquímica em 39 casos e destes $66,6 \%$ foram positivos.

A pesquisa de Her2/neu nos carcinomas invasivos mostrou hiperexpressão em $61,7 \%$ dos casos, foi mais incidente nos tumores maiores que $2 \mathrm{~cm}(34,6 \%)$ e o grau II histológico foi o que apresentou maior hiperexpressão desta (42,3\%). Estes dados corroboram os dados da literatura que mostram que a hiperexpressão do Her2 está relacionada à maior grau histológico, aumento da agressividade tumoral, redução da sobrevida e aumento da recorrência e mortalidade (JIANG et al., 2012), embora não tenha sido observada relação estatisticamente significante para hiperexpressão desta proteína e índice mitótico.

O status patológico dos linfonodos no câncer de mama é classificado como negativo, 1 a 3 linfonodos positivos e 4 ou mais linfonodos positivos. Tanto o status negativo e 01 a 3 linfonodos positivos são considerados de risco intermediário se combinados com hiperexpressão de Her2 (TAKEl et al., 2007). Em apenas cinco casos com invasão angio-linfática observou-se 
hiperexpressão de Her2/neu e na maioria (46,1\%) daqueles com hiperexpressão de Her2/neu não ocorreu metástase para linfonodos axilares.

A positividade para Her2 está associada com um risco aumentado de falha em qualquer terapia endócrina (RASTELLI; CRISPINO, 2008). Em 50\% dos casos positivos para RE/RP houve hiperexpressão do Her2/neu concomitante, mostrando que apesar de muitos casos terem tido diagnóstico precoce e o fato de serem positivos para RE/RP fosse de bom prognóstico para as pacientes, a hiperexpressão de Her2/neu pode interferir no prognóstico e no tratamento adjuvante destas pacientes.

Nossos dados corroboram que o status patológico dos linfonodos axilares, a graduação do tumor, o tamanho tumoral e o status do RE são os fatores mais importantes, clinicamente, a serem considerados na seleção das mulheres com câncer de mama precoce para realização de terapia adjuvante (WILLIANS et al., 2006).

\section{CONCLUSÃO}

Diante dos resultados obtidos, conclui-se que houve baixa prevalência de câncer de mama na população estudada e que a maioria teve diagnóstico precoce para o câncer de mama, o que melhorou o prognóstico das pacientes. Porém, em poucas pacientes foi realizada a avaliação do status dos receptores hormonais e da proteína Her2/neu, o que é de suma importância para adequada avaliação do prognóstico e da resposta a terapêuticas específicas para o câncer de mama.

\section{REFERÊNCIAS}

Brasil. Ministério da Saúde. Secretaria de Atenção a Saúde. Instituto Nacional do Câncer. Estimativa 2012: Incidência de câncer no Brasil. Rio de Janeiro: INCA, 2011.

Brasil. Ministério da Saúde. Secretaria de Atenção a Saúde. Instituto Nacional do Câncer. Controle do câncer de mama - documento de consenso. Rio de Janeiro: INCA, 2004.
Brot M, Soares FA, Stiepcich MA, Curcio VS, Gobbi H. Carcinomas mamários de tipo basal: perfil clinico-patologico e evolutivo. Revista da Associação Médica Brasileira 2009; 55(5):529-34. DOI: $\quad$ http://dx.doi.org/10.1590/S010442302009000500014

Elston CW, Ellis IO. Pathological prognostic factors in breast cancer. I. The value of histological grade in breast cancer: experience from a large study with long-term follow-up. Histopathology 2002;41(3A):154-61. DOI: http://dx.doi.org/10.1046/j.13652559.2002.14691.x

Faneyte IF, Peterse JL, van Tinteren H, Pronk C, de Vries EGE, Rodenhuis $S$, van de Vijver $M J$. Predicting early failure after adjuvant chemotherapy in high-risk breast cancer patients with extensive lymph node involvement. Clinical Cancer Research 2004; 10. DOI: http://dx.doi.org/10.1158/1078-0432.CCR-0300544457-63.

Gatek J, Vrana D, Hnatek L, Bakala J, Dudesek B, Duben J, Musil T. Sentinel node biopsy and neoadjuvant chemotherapy in the treatment of breast cancer. Journal of the Balkan Union of Oncology 2012;17(2):265-70.

Jiang $\mathrm{H}$, Bai $\mathrm{X}$, Zhang $\mathrm{C}$, Zhang $\mathrm{X}$. Evaluation of HER2 gene amplification in breast cancer using nuclei microarray in situ hybridization. International Journal of Molecular Sciences. 2012; 13: 5519-27. DOI: http://dx.doi.org/10.3390/ijms13055519

Kurosumi M. Significance of immunohistochemical assessment of steroid hormone receptor status for breast cancer patients. Breast Cancer. 2003;10(2):97-104.

DOI: http://dx.doi.org/10.1007/BF02967633

Nai GA, Fleury A, Jacob MFFB, Silva EGA, Barbosa RL. Diagnóstico mamográfico de microcalcificações suspeitas para malignidade e sua correlação com os achados anatomopatológicos. Revista Brasileira de Clínica e Terapêutica 2006; 32(3): 134-8.

Nai GA, Vilhegas RB, Jacob MFFB, Martins JF, Lopes CCB, Oliveira KS. Projeto de extensão universitária de orientação sobre o câncer de mama. Revista Brasileira de Medicina 2009; 66: 342-6.

Paulinelli RR, Junior RF, Curado MP, Souza AA. A situação do câncer de mama em Goiás, no Brasil e no mundo: tendências atuais para a incidência e mortalidade. Revista Brasileira de Saúde Materno Infantil 2003; 3(1):17-24. DOI: http://dx.doi.org/10.1590/S151938292003000100004 
Payne SJ, Bowen RL, Jones JL, Wells CA.

Predictive markers in breast cancer--the present. Histopathology. 2008;52(1):82-90. DOI: http://dx.doi.org/10.1111/j.13652559.2007.02897.x

Rastelli F, Crispino S. Factors predictive of response to hormone therapy in breast cancer. Tumori. 2008;94(3):370-83.

Reyal F, Bollet MA, Caly M, Gentien D, Carpentier S, Peyro-Saint-Paul HH, Pierga J-Y, Cottu Paul, Dieras V, Sigal-Zafrani B, Vincent-Salomon A, Sastre-Garau X. Respective Prognostic Value of Genomic Grade and Histological Proliferation Markers in Early Stage (pNO) Breast Carcinoma. PLOS ONE 2012; 7(4): e35184. DOI: http://dx.doi.org/10.1371/journal.pone.0035184

Rivadeneira DE, Simmons RM, Christos PJ, Hanna K, Daly JM, Osborne MP. Predictive factors associated with axillary lymph node metastases in T1a and T1b breast carcinomas: analysis in more than 900 patients. The Journal of the American College of Surgeons 2000;191(1):1-6. DOI: http://dx.doi.org/10.1016/S1072-7515(00)00310-0

Sclowitz ML, Menezes AMB, Gigante DP, Tessaro S. Condutas na prevenção secundaria do câncer de mama e fatores associados. Revista de Saúde Pública 2005; 39(3):340-9. DOI: http://dx.doi.org/10.1590/S003489102005000300003

Song $\mathrm{YJ}$, Shin $\mathrm{SH}$, Cho JS, Park $\mathrm{MH}$, Yoon JH, Jegal $Y$. The role of lymphovascular invasion as a prognostic factor in patients with lymph nodepositive operable invasive breast cancer. Journal of Breast Cancer 2011; 14(3):198-203. DOI: http://dx.doi.org/10.4048/jbc.2011.14.3.198

Takei H, Kurosumi M, Yoshida T, Ninomiya J, Hagiwara Y, Kamimura M, Hayashi H, Tozuka K, Suemasu K, Inoue K, Tabei T. Current trends of sentinel lymph node biopsy for breast cancer - a surgeon's perspective. Breast Cancer 2007;14:362-70.

DOI:

http://dx.doi.org/10.2325/jbcs.14.362

Vieira DSC, Dufloth RM, Schmitt FCL, Zeferino LC. Carcinoma de mama: novos conceitos na classificação, Revista Brasileira de Ginecologia e Obstetrícia 2008; 30(1):42-7. DOI: http://dx.doi.org/10.1590/S0100-

72032008000100008

Williams C, Brunskill S, Altman D, Briggs A, Campbell H, Clarke M, Glanville J, Gray A, Harris A, Johnston K, Lodge M. Cost-effectiveness of using prognostic information to select women with breast cancer for adjuvant systemic therapy. 